\title{
Dynamical Analysis of Transmission of Hepatitis B and $C$ Viruses with External Source of disease by Mathematical Model
}

\author{
Ahmed Mohsen ( $\square$ aamuhseen@gmail.com ) \\ University of Baghdad https://orcid.org/0000-0003-3812-8918 \\ Khalid Hattaf \\ Université Hassan II de Casablanca: Universite Hassan II de Casablanca \\ Hassan AL-Husseiny \\ University of Baghdad Al-Jaderyia Campus College of Science
}

\section{Research Article}

Keywords: Epidemic Model, Hepatitis B and C Viruses, Vaccination, External Sources of infection, Vertical and Horizontal transmission, Stability

Posted Date: September 7th, 2021

DOI: https://doi.org/10.21203/rs.3.rs-730763/v1

License: (9) This work is licensed under a Creative Commons Attribution 4.0 International License. Read Full License 


\title{
Dynamical Analysis of Transmission of Hepatitis B and C Viruses with External Source of disease by Mathematical Model
}

\author{
The Hepatitis B and C Viruses with External Source of \\ disease
}

\author{
Ahmed A. Mohsen • Khalid Hattaf • \\ Hassan Fadhil AL-Husseiny
}

Received: date / Accepted: date

\begin{abstract}
In this paper, we develop a deterministic mathematical model of the Hepatitis $\mathrm{B}$ and $\mathrm{C}$ viruses transmission in population, which allows transmission by two way vertical such as from pregnant mother to fetus and horizontal. Also, by two way through direct contact and due to the external source of infective such as blood transfusion or other. In reality, we know that there is a vaccination against the hepatitis $B$ virus but so far, there is no vaccine against the hepatitis $C$ virus this is why it is considered more dangerous than hepatitis B. Furthermore, we study the vaccination effect with the failure in the vaccine. We propose an $S V I_{B} I_{C} R$ model using a system of ordinary differential equations. First the major basic analysis, like the uniqueness, boundedness and positivity of the solution for the proposed model. Second the existence of all biological equilibrium points, basic reproduction number and stability analysis of all equilibrium points. The numerical simulation indicated to confirm the analytic results and the government must apply all control strategies in combating hepatitis virus at short periods of time.
\end{abstract}

Keywords Epidemic Model · Hepatitis $B$ and $C$ Viruses · Vaccination; External Sources of infection - Vertical and Horizontal transmission . Stability.

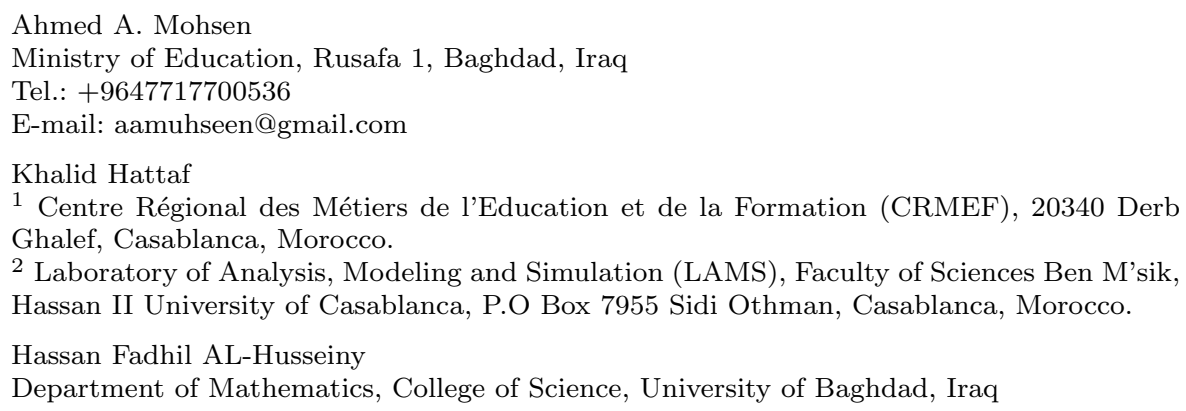


Mathematics Subject Classification (2020) 92Bxx - 03C45 - 34Dxx · $32 \mathrm{Cxx} \cdot 00 \mathrm{~A} 71$.

\section{Introduction}

There are several types of viruses that infect humans and cause many diseases, such as Encephalitis, HPV (Human papilloma virus), HIV, Yellow fever, Impetigo, Poliomyelitis, Chicken pox, Measles, Mumps, HBV (hepatitis B virus), HCV (hepatitis C virus) and lastly COVID-19. In fact, viral hepatitis is considered one of the serious global epidemic diseases caused by HBV. It mainly attacks the liver, it may be a chronic disease or lead to death and sometimes it turns into liver cancer. According to reports from the World Health Organization (WHO) in 2020, about 240 million people in the world are living with chronic liver infection and approximately 0.78 million died. While, in another reports in 2017, the hepatitis affects the lives of 257 million people and death 56000 people every year $[1,2]$.

According to the mechanism of transmission of hepatitis, it is one of the infectious diseases that is transmitted from one person to another, and the most transmissible method is sexual intercourse through semen, blood and vaginal excretion as well as by external sources of infective for example sharing unhygienic needles or razors, transfusion of infected human blood, tattoo and dialysis machines, therefor all above ways to spreading is called horizontal transmissions. Also, this infection transmits from mother to newborn baby at the time of birth but called the vertical transmission [3-5]. Since there is no effective treatment yet, the vaccination that was discovered in 1982, is very important and is necessary today because infection with hepatitis has a high mortality rate. The vaccine protects children from serious infection with the hepatitis and enhances the immune system against infection [6].

There are five primary types of hepatitis, which are A, B, C, D and E. The types $\mathrm{A}$ and $\mathrm{E}$ come through contamination of food or water, and not turn into chronic hepatitis and are completely cured of them and do not leave traces of the liver. As for the other three B, C and D, they are come through blood and its derivatives, and sexual relations and them turns into chronic liver infections that and leave an impact and damage to the liver.

Some related literature and works regarding the Hepatitis are considered in [7]-[11]. In addition, Gul et al. [12] studied symptomatic carrier effect on the dynamics of hepatitis B virus. Saad et al. [13] studied hepatitis C virus by fractional order modeling. The authors in [14] proposed a new mathematical model for the transmission of HBV due to sexual. Khatun et al. [15] introduced a mathematical modeling of hepatitis B virus with immune responses. In [16], Din et al. formulated a model of hepatitis B epidemic with stochastic analysis and delay effect. $\mathrm{Lu}$ et al. [17] studied and analyzed a modeling of hepatitis transmission in China through homosexual and heterosexual. In this paper, we formulate a mathematical model to analyze the infection transmission of hepatitis B and C viruses. Different from previous studies of hepatitis mod- 


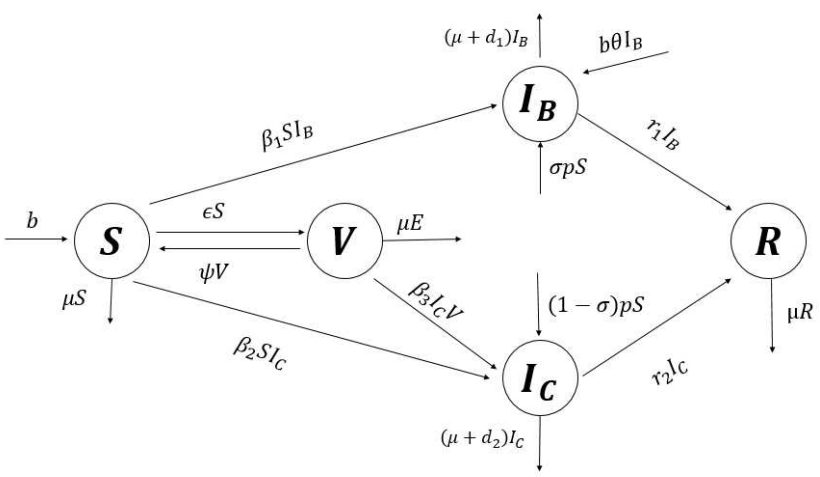

Fig. 1 Diagram of Hepatitis Model.

els, we focus on the vertical transmission of hepatitis B which does not exist in hepatitis C. As well as, we discuss the failure of the vaccination against hepatitis B virus and the risk of not being available against hepatitis C. This work is ordered as follows. The model divided the population into five groups is formulated, and then the basic properties of solutions of the proposed model such as uniqueness, boundedness and positivity are investigated. Also, we calculate the basic reproduction number of the model. Finally, we analyze and study the local and global stability about the all four equilibrium points of the model.

\section{Model Formulation}

In this section, we present the proposed mathematical model of HBV and $\mathrm{HCV}$ infection disease. To create the model, we assume that the total human population $N(t)$ is divided into five epidemiological sub-compartments namely and denoted by susceptible $S(t)$; vaccination $V(t)$; infected by hepatitis $B$ virus $I_{B}(t)$; infected by hepatitis $C$ virus $I_{C}(t)$ and recovered $R(t)$ respectively. Such that $N(t)=S(t)+V(t)+I_{B}(t)+I_{C}(t)+R(t)$. The transmission dynamics of $\mathrm{HBV}$ and HCV is given by the following diagram of model and the nonlinear differential equations.

$$
\begin{aligned}
& \dot{S}=b\left(1-\theta I_{B}\right)-\left(\mu+\epsilon+p+\beta_{1} I_{B}+\beta_{2} I_{C}\right) S+\psi V, \\
& \dot{V}=\epsilon S-\left(\mu+\psi+\beta_{3} I_{C}\right) V, \\
& \dot{I_{B}}=\left(b \theta+\beta_{1} S-\mu-d_{1}-r_{1}\right) I_{B}+\sigma p S, \\
& \dot{I_{C}}=\left(\beta_{2} S+\beta_{3} V-\mu-d_{2}-r_{2}\right) I_{C}+(1-\sigma) p S, \\
& \dot{R}=r_{1} I_{B}+r_{2} I_{C}-\mu R,
\end{aligned}
$$

with the initial conditions for model (1) satisfy:

$$
S(0)>0, V(0)>0, I_{B}(0) \geq 0, I_{C}(0) \geq 0, R(0) \geq 0 .
$$


In above model, recruitment rate is $b$ and the natural death in all population is $\mu$. The horizontal transmission of disease by contact rates and denoted by $\beta_{i}, i=1,2,3$. For vertical transmission, we assume that a fraction $\theta$ of newborns from infected by hepatitis $\mathrm{B}$ virus class are infected and it is denoted by $(0 \leq \theta \leq 1)$. The both hepatitis $\mathrm{B}$ and $\mathrm{C}$ are also spread by external sources denoted by $p \geq 0$ with fraction $(0 \leq \sigma \leq 1)$. The susceptible class is vaccinated at per capita rate $\epsilon>0$. The vaccine provides temporary immunity (failure in vaccine), that is denoted by $(0 \leq \psi \leq 1)$. The disease mortality rates in the HBV and HCV are $d_{1}$ and $d_{2}$, respectively. The HBV and HCV individuals are recovered at the rates $r_{1}$ and $r_{2}$, respectively.

\section{Basic Properties of Solutions}

The hepatitis model (1), will be meaningful epidemiologically if the solutions of (1) with non-negative initial conditions (2) will remain non-negative for all time $t>0$.

In this section, we investigate the existence, uniqueness, boundedness and positivity of the solution in the following theorems.

\subsection{Existence and uniqueness}

Theorem 3.1 There exists a unique solution of model (1), in a positively invariant set, that remains for all finite time $t \geq 0$.

Proof. The right-hand side of all equations is continuous in the convex domain $E=\left(t, S(t), V(t), I_{B}(t), I_{C}(t), R(t)\right)$ of $(5+1)$-dimensional space $\Re_{+}^{5+1}$, with continuous partial derivatives. So problem (1) has a unique solution in $\Re_{+}^{5}$, which exists for a given finite time $t \in[0,+\infty)$ and initial conditions (2).

\subsection{Boundedness}

Theorem 3.2 All solutions of model (1) which initiate in $\Re_{+}^{5}$, are uniformly bounded.

Proof. As the total population size are $N=S+V+I_{B}+I_{C}+R$, so from model (1) we get

$$
\dot{N}=b-\mu N-d_{1} I_{B}-d_{2} I_{C} .
$$

Then $\dot{N} \leq b-\mu N$. Hence, $\mathrm{N}(\mathrm{t}) \leq N(0) e^{-\mu t}+\frac{b}{\mu}\left(1-e^{-\mu t}\right)$, which shows that

$$
N(t) \leq \max \left\{N(0), \frac{b}{\mu}\right\}
$$

This implies the the boundedness of solutions. 


\subsection{Positivity}

Theorem 3.3 All solutions $S(t), V(t), I_{B}(t), I_{C}(t), R(t)$ of model (1), starting from positive initial conditions (2), remain positive for all finite time $t \geq 0$.

Proof. We have

$\left.\dot{S}\right|_{S=0}=b\left(1-\theta I_{B}\right)+\psi V>0$, forall $I_{B}, V \geq 0$,

$\left.\dot{V}\right|_{V=0}=\epsilon S>0$, forall $S>0$,

$\left.\dot{I_{B}}\right|_{I_{B}=0}=\sigma p S \geq 0$, forall $S>0$,

$\left.\dot{I_{C}}\right|_{I_{C}=0}=(1-\sigma) p S \geq 0$, forall $S>0$,

$\left.\dot{R}\right|_{R=0}=r_{1} I_{B}+r_{2} I_{C} \geq 0$, forall $I_{B}, I_{C} \geq 0$.

This leads to the non-negativity of solutions.

On the other hand, since the 5 th equation of model (1), is independent on the other equations rest, so model (1) we can be reduced to the following system:

$$
\begin{aligned}
& \dot{S}=b\left(1-\theta I_{B}\right)-\left(\mu+\epsilon+p+\beta_{1} I_{B}+\beta_{2} I_{C}\right) S+\psi V, \\
& \dot{V}=\epsilon S-\left(\mu+\psi+\beta_{3} I_{C}\right) V, \\
& \dot{I_{B}}=\left(b \theta+\beta_{1} S-\mu-d_{1}-r_{1}\right) I_{B}+\sigma p S, \\
& \dot{I_{C}}=\left(\beta_{2} S+\beta_{3} V-\mu-d_{2}-r_{2}\right) I_{C}+(1-\sigma) p S .
\end{aligned}
$$

4 Existence of equilibrium point and basic reproduction number of model (5)

Obviously, model (5) has a disease free equilibrium point when $I_{B}=I_{C}=0$ with $p=0$ and denoted by $e_{0}=\left(S_{0}, V_{0}, 0,0\right)$, where

$$
\left\{\begin{array}{l}
S_{0}=\frac{b[\mu(\mu+\epsilon+\psi)+\epsilon \psi]}{\mu(\mu+\epsilon)(\mu+\epsilon+\psi)}, \\
V_{0}=\frac{\epsilon b}{\mu(\mu+\epsilon+\psi)} .
\end{array}\right.
$$

Then we define the basic reproduction number $\mathcal{R}_{0}$ of the model (5) is obtained by using the next generation technique [18], and is given by

$$
\mathcal{R}_{0}=\mathcal{R}_{0 B}+\mathcal{R}_{0 C}
$$

where

$-\mathcal{R}_{0 B}=\frac{b \theta \mu(\mu+\epsilon)(\mu+\epsilon+\psi)+b \beta_{1}[\mu(\mu+\epsilon+\psi)+\epsilon \psi]}{\mu(\mu+\epsilon)(\mu+\epsilon+\psi)\left(\mu+d_{1}+r_{1}\right)}$, is the basic reproduction number for HBV infected.

$-\mathcal{R}_{0 C}=\frac{b \beta_{2}[\mu(\mu+\epsilon+\psi)+\epsilon \psi]+\beta_{3} \epsilon b(\mu+\epsilon)}{\mu(\mu+\epsilon)(\mu+\epsilon+\psi)\left(\mu+d_{2}+r_{2}\right)}$,

is the basic reproduction number for $\mathrm{HCV}$ infected.

Now, model (5) has a hepatitis $B$ virus-free equilibrium point when $I_{B}=0$ under the condition $\sigma=0$ and denoted by $e_{1}=\left(S_{1}, V_{1}, 0, I_{C 1}\right)$, where.

$$
\left\{\begin{array}{l}
S_{1}=\frac{b\left(\mu+\psi+\beta_{3} I_{C 1}\right)}{\left(\mu+\beta_{3} I_{C 1}\right)\left(\mu+\epsilon+p+\beta_{2} I_{C 1}\right)+\psi\left(\mu+p+\beta_{2} I_{C 1}\right)}, \\
V_{1}=\frac{\epsilon b}{\left(\mu+\beta_{3} I_{C 1}\right)\left(\mu+\epsilon+p+\beta_{2} I_{C 1}\right)+\psi\left(\mu+p+\beta_{2} I_{C 1}\right)}
\end{array}\right.
$$


Also, we can calculated the positive value of $I_{C 1}$ from the polynomial as follow

$$
A_{1} I_{C 1}^{3}+A_{2} I_{C 1}^{2}+A_{3} I_{C 1}+A_{4}=0
$$

here

$A_{1}=-\beta_{2} \beta_{3}\left(\mu+d_{2}+r_{2}\right)<0$

$A_{2}=b \beta_{2} \beta_{3}-\left(\mu+d_{2}+r_{2}\right)\left(\mu \beta_{2}+\beta_{3}(\mu+\epsilon+p)+\psi \beta_{2}\right)$,

$A_{3}=b \beta_{3}(\epsilon+p)-\left[\mu(\mu+\epsilon+p)+\psi(\epsilon+p)\left(\mu+d_{2}+r_{2}\right)\right]$,

$A_{4}=b p \beta_{3}>0$.

Then, we have a unique positive root of $I_{C 1}$ in equation (9), if and only if $A_{2}<0$ or $A_{3}>0$.

Clearly, model (5) has a hepatitis $C$ virus-free equilibrium point when $I_{C}=0$ under the condition $\sigma=1$ and denoted by $e_{2}=\left(S_{2}, V_{2}, I_{B 2}, 0\right)$, where.

$$
\left\{\begin{array}{l}
S_{2}=\frac{b\left(1-\theta I_{B 2}\right)(\mu+\psi)}{\mu\left(\mu+\epsilon+p+\beta_{1} I_{B 2}\right)+\psi\left(\mu+p+\beta_{1} I_{B 2}\right)}, \\
V_{2}=\frac{b\left(1-\theta I_{B 2}\right)}{\mu\left(\mu+\epsilon+p+\beta_{1} I_{B 2}\right)+\psi\left(\mu+p+\beta_{1} I_{B 2}\right)}
\end{array}\right.
$$

It easy see that, the all values of $S_{2}$ and $V_{2}$ became a positive under the following condition

$$
\theta I_{B 2}<1
$$

While, we can calculated the positive value of $I_{C 1}$ from the polynomial as follow

$$
B_{1} I_{B 2}^{2}+B_{2} I_{B 2}+B_{3}=0
$$

here

$B_{1}=-\beta_{1}(\mu+\psi)\left(\mu+d_{1}+r_{1}\right)<0$,

$B_{2}=b\left[\mu \theta(\mu+\epsilon+p)+\psi \theta(\mu+p)+\beta_{1}(\mu+\psi)\right]-b p \theta(\mu+\psi)-\left(\mu+d_{1}+r_{1}\right)[\psi(\mu+p)+\mu(\mu+\epsilon+p)]$, $B_{3}=b p(\mu+\psi)>0$.

Then, we have a unique positive root of $I_{B 2}$ in equation (12), if and only if $B_{2}<0$ or $B_{2}>0$.

Lastly, model (5) has the endemic equilibrium point and denoted by $e_{3}=$ $\left(S_{3}, V_{3}, I_{B 3}, I_{C 3}\right)$, where.

$$
\left\{\begin{array}{l}
S_{3}=\frac{b\left(1-\theta I_{B 3}\right)\left(\mu+\psi+\beta_{3} I_{C 3}\right)}{\left(\mu+\psi+p+\beta_{3} I_{C 3}\right)\left(\mu+p+\beta_{1} I_{B 3}+\beta_{2} I_{C 3}\right)+\epsilon\left(\mu+\beta_{3} I_{C 3}\right.}, \\
V_{3}=\frac{\epsilon b\left(1-\theta I_{B 3}\right)}{\left(\mu+\psi+p+\beta_{3} I_{C 3}\right)\left(\mu+p+\beta_{1} I_{B 3}+\beta_{2} I_{C 3}\right)+\epsilon\left(\mu+\beta_{3} I_{C 3}\right.} .
\end{array}\right.
$$

While $\left(I_{B 3}, I_{C 3}\right)$ represents the positive intersection point of the following two isoclines

$$
\begin{aligned}
f\left(I_{B}, I_{C}\right)=\{ & \left.\left(b \theta-\mu-d_{1}-r_{1}\right)\left[\left(\mu+\psi+\beta_{3} I_{C}\right)\left(\mu+p+\beta_{1} I_{B}+\beta_{2} I_{C}\right)+\epsilon\left(\mu+\beta_{3} I_{C}\right)\right]\right\} I_{B} \\
& +b\left(\beta_{1} I_{B}\right)\left(1-\theta I_{B}\right)\left(\mu+\psi+\beta_{3} I_{C}\right)=0,
\end{aligned}
$$


Now, if $I_{C} \rightarrow 0$, we get the isoclines becomes

$$
f\left(I_{B}\right)=C_{1} I_{B}^{2}+C_{2} I_{B}+C_{3}=0
$$

where

$C_{1}=-\beta_{1}(\mu+\psi)\left(\mu+d_{1}+r_{1}\right)<0$,

$C_{2}=\left(b \theta-\mu-r_{1}-d_{1}\right)[(\mu+\psi)(\mu+p)+\epsilon \mu]+b(\mu+\psi)\left(\beta_{1}-\sigma p \theta\right)$,

$C_{3}=b p \sigma(\mu+\psi)>0$.

$$
g\left(I_{B}\right)=b p(\mu+\psi)(1-\sigma)\left(1-\theta I_{B}\right)=0 .
$$

Then, equation (16) intersects the $I$-axis at the positive $\widetilde{I_{B}}$, while equation (17) intersects the $I$-axis at the positive $\widehat{I_{B}}=\frac{1}{\theta}$. Clearly, the equations (14) and (15) have a unique positive intersection point and then $e_{3}$ exists uniquely, under the following conditions

$$
\begin{gathered}
\left\{\begin{array}{c}
C_{2}<0, \\
o r \quad \\
C_{2}>0,
\end{array}\right. \\
\widehat{I_{B}}<\widetilde{I_{B}}, \\
\left\{\begin{array}{l}
\frac{d I_{B}}{d I_{C}}=-\frac{\partial f / \partial I_{C}}{\partial f / \partial I_{B}}<0, \\
\frac{d I_{B}}{d I_{C}}=-\frac{\partial g / \partial I_{C}}{\partial g / \partial I_{B}}>0 .
\end{array}\right.
\end{gathered}
$$

\section{Local Stability Analysis}

In the following of this section, we investigate the local stability of model (5) around all equilibrium points using the linearization method. It easy we can calculating the general Jacobian matrix of model (5) about an arbitrary point $e^{*}=\left(S, V, I_{B}, I_{C}\right)$ and can be written in below

$J\left(e^{*}\right)=\left(\begin{array}{cccc}-\left(\mu+\epsilon+p+\beta_{1} I_{B}+\beta_{2} I_{C}\right) & \psi & -\left(b \theta+\beta_{1} S\right) & -\beta_{2} S \\ \epsilon & -\left(\mu+\psi+\beta_{3} I_{C}\right) & 0 & -\beta_{3} V \\ \beta_{1} I_{B}+\sigma p & 0 & b \theta+\beta_{1} S-\left(\mu+d_{1}+r_{1}\right) & 0 \\ \beta_{2} I_{C}+(1-\sigma) p & \beta_{3} I_{C} & 0 & \beta_{2} S+\beta_{3} V-\left(\mu+d_{2}+r_{2}\right)\end{array}\right)$.

Now, rewrite the equation (21) around a disease free equilibrium point $e_{0}$ has the following result

$$
J\left(e_{0}\right)=\left(\begin{array}{cccc}
-(\mu+\epsilon) & \psi & -\left(b \theta+\beta_{1} S_{0}\right) & -\beta_{2} S_{0} \\
\epsilon & -(\mu+\psi) & 0 & -\beta_{3} V_{0} \\
0 & 0 & b \theta+\beta_{1} S_{0}-\left(\mu+d_{1}+r_{1}\right) & 0 \\
0 & 0 & 0 & \beta_{2} S_{0}+\beta_{3} V_{0}-\left(\mu+d_{2}+r_{2}\right)
\end{array}\right) .
$$


Then, the characteristic polynomial of matrix (22) is

$\left[b \theta+\beta_{1} S_{0}-\left(\mu+d_{1}+r_{1}\right)-\lambda\right]\left[\beta_{2} S_{0}+\beta_{3} V_{0}-\left(\mu+d_{2}+r_{2}\right)-\lambda\right]\left[\lambda^{2}+T_{0} \lambda+D_{0}\right]=0$,

here

$T_{0}=2 \mu+\epsilon+\psi>0$,

$D_{0}=\mu(\mu+\psi+\epsilon)>0$.

Consequently, equation (23) has following roots, which represent the eigenvalues of matrix $(22)$

$\left\{\begin{array}{l}\lambda_{S}=-(\mu+\epsilon+\psi), \\ \lambda_{V}=-\mu, \\ \lambda_{I_{B}}=b \theta+\beta_{1} S_{0}-\left(\mu+d_{1}+r_{1}\right), \\ \lambda_{I_{C}}=\beta_{2} S_{0}+\beta_{3} V_{0}-\left(\mu+d_{2}+r_{2}\right) .\end{array}\right.$

Clearly, $\lambda_{S}$ and $\lambda_{V}$ have always negative real parts, while $\lambda_{I_{B}}$ and $\lambda_{I_{C}}$ are negative real parts when $\mathcal{R}_{0}<1$. Hence, $e_{0}$ is locally asymptotically stable. However, it is a saddle point otherwise.

Again, rewrite the equation (21) but around a hepatitis $B$ virus-free equilibrium point $e_{1}$ has the following result

$$
J\left(e_{1}\right)=\left(\begin{array}{cccc}
m_{11} & m_{12} & m_{13} & m_{14} \\
m_{21} & m_{22} & 0 & m_{24} \\
0 & 0 & m_{33} & 0 \\
m_{41} & m_{42} & 0 & m_{44}
\end{array}\right)
$$

Here

$$
\begin{gathered}
m_{11}=-\left(\mu+\epsilon+p+\beta_{2} I_{C 1}\right) ; m_{12}=\psi ; m_{13}=-\left(b \theta+\beta_{1} S_{1}\right) ; m_{14}=-\beta_{2} S_{1} \\
m_{21}=\epsilon ; m_{22}=-\left(\mu+\psi+\beta_{3} I_{C 1}\right) ; m_{24}=-\beta_{3} V_{1} \\
m_{33}=b \theta+\beta_{1} S_{1}-\left(\mu+d_{1}+r_{1}\right) ; m_{41}=\beta_{2} I_{C 1}+p ; m_{42}=\beta_{3} I_{C 1} \\
m_{44}=\beta_{2} S_{1}+\beta_{3} V_{1}-\left(\mu+d_{2}+r_{2}\right) ; m_{23}=m_{31}=m_{23}=m_{34}=m_{43}=0 .
\end{gathered}
$$

Then, the characteristic polynomial of matrix (24) is

$$
\left[b \theta+\beta_{1} S_{1}-\left(\mu+d_{1}+r_{1}\right)-\lambda\right]\left[\lambda^{3}+M_{1} \lambda^{2}+M_{2} \lambda+M_{3}\right]=0
$$

where

$$
\begin{aligned}
& M_{1}=-\left(m_{11}+m_{22}+m_{44}\right), \\
& M_{2}=\left(m_{11} m_{22}-m_{12} m_{21}+m_{11} m_{44}-m_{14} m_{41}+m_{22} m_{44}-m_{24} m_{42}\right), \\
& M_{3}=-\left(m_{44}\left(m_{11} m_{22}-m_{12} m_{21}\right)+m_{41}\left(m_{12} m_{24}-m_{22} m_{14}\right)+m_{42}\left(m_{21} m_{14}-m_{11} m_{24}\right)\right) .
\end{aligned}
$$

Obviously, one of the eigenvalues of $b \theta+\beta_{1} S_{1}-\left(\mu+d_{1}+r_{1}\right)$ in equation (25) can has negative real part if $m_{33}<0$. The remaining eigenvalues can be obtained from the cubic equation (25). Clearly, if it satisfies the Routh Hurtwiz criterion conditions that $M_{i}>0$ for $i=1,3$ and $\Delta=M_{1} M_{2}-M_{3}>$ 0 . So, $M_{i}>0$ for $i=1,3$ with $\Delta>0$ can be easily confirm if and only if $m_{44}<0$ and $m_{11} m_{22}>m_{12} m_{21}$. Therefore, the $e_{1}$ of the model (5) is locally asymptotically stable. 
Now, the equation (21) around a hepatitis $C$ virus-free equilibrium point $e_{2}$ can be written as following

$$
J\left(e_{2}\right)=\left(\begin{array}{cccc}
w_{11} & w_{12} & w_{13} & w_{14} \\
w_{21} & w_{22} & 0 & w_{24} \\
w_{31} & 0 & w_{33} & 0 \\
0 & 0 & 0 & w_{44}
\end{array}\right) .
$$

Here

$$
\begin{gathered}
w_{11}=-\left(\mu+\epsilon+p+\beta_{1} I_{B 1}\right) ; w_{12}=\psi ; w_{13}=-\left(b \theta+\beta_{1} S_{2}\right) ; w_{14}=-\beta_{2} S_{2} \\
w_{21}=\epsilon ; w_{22}=-(\mu+\psi) ; w_{24}=-\beta_{3} V_{2} \\
w_{31}=\beta_{1} I_{B 1}+p ; \quad w_{33}=b \theta+\beta_{1} S_{2}-\left(\mu+d_{1}+r_{1}\right) ; w_{44}=\beta_{2} S_{2}+\beta_{3} V_{2}-\left(\mu+d_{2}+r_{2}\right) \\
w_{23}=w_{32}=w_{34}=w_{41}=w_{42}=w_{43}=0 .
\end{gathered}
$$

Then the characteristic polynomial of matrix $(26)$ is

$$
\left[\beta_{2} S_{2}+\beta_{3} V_{2}-\left(\mu+d_{2}+r_{2}\right)-\lambda\right]\left[\lambda^{3}+W_{1} \lambda^{2}+W_{2} \lambda+W_{3}\right]=0,
$$

where

$W_{1}=-\left(w_{11}+w_{22}+w_{33}\right)$,

$W_{2}=\left(w_{11} w_{22}-w_{12} w_{21}+w_{11} w_{33}-w_{13} w_{31}+w_{22} w_{33}\right)$,

$W_{3}=-\left(w_{33}\left(w_{11} w_{22}-w_{12} w_{21}\right)-w_{22} w_{13} w_{31}\right)$.

Clearly, in equation (27) one of the eigenvalues is $\beta_{2} S_{2}+\beta_{3} V_{2}-\left(\mu+d_{2}+r_{2}\right)$ has negative real part if $w_{44}<0$. The other eigenvalues can be find from the cubic equation (27). Clearly, if it satisfies the Routh-Hurtwiz criterion conditions that $W_{i}>0$ for $i=1,3$ and $\Delta=W_{1} W_{2}-W_{3}>0$. So, $W_{i}>0$ for $i=1,3$ with $\Delta>0$ can be easily confirm if and only if $w_{33}<0$ and $w_{11} w_{22}>w_{12} w_{21}$. Therefore, the $e_{2}$ of the model (5) is locally asymptotically stable.

Finally, about endemic equilibrium point $e_{3}$ the equation (21) becomes below

$$
J\left(e_{3}\right)=\left(\begin{array}{cccc}
q_{11} & q_{12} & q_{13} & q_{14} \\
q_{21} & q_{22} & 0 & q_{24} \\
q_{31} & 0 & q_{33} & 0 \\
q_{41} & q_{42} & 0 & q_{44}
\end{array}\right) .
$$

Here

$$
\begin{gathered}
q_{11}=-\left(\mu+\epsilon+p+\beta_{1} I_{B 3}+\beta_{2} I_{C 3}\right) ; q_{12}=\psi ; q_{13}=-\left(b \theta+\beta_{1} S_{3}\right) \\
q_{14}=-\beta_{2} S_{3} q_{21}=\epsilon ; q_{22}=-\left(\mu+\psi+\beta_{3} I_{C 3}\right) ; q_{24}=-\beta_{3} V_{3} \\
q_{31}=\beta_{1} I_{B 3}+\sigma p ; q_{33}=b \theta+\beta_{1} S_{3}-\left(\mu+d_{1}+r_{1}\right) \\
q_{41}=\beta_{2} I_{C 3}+(1-\sigma) p ; q_{42}=\beta_{3} I_{C 3} ; q_{44}=\beta_{2} S_{3}+\beta_{3} V_{3}-\left(\mu+d_{2}+r_{2}\right) .
\end{gathered}
$$

Obviously, according to Gersgorin theorem [19] if the condition $\left|q_{i i}\right|>$ $\sum_{i=1, i \neq j}^{4}\left|q_{i j}\right|$, holds. Then the all eigenvalues of matrix (28) can be obtained in the left half plane and exists in the region $\Lambda=\cup\left\{v^{*} \in \Theta:\left|v^{*}-q_{i i}\right|<\right.$ $\left.\sum_{i=1, i \neq j}^{4}\left|q_{i j}\right|\right\}$, where $\Lambda$ represents the union of all the discs centered at $q_{i i}$ with radius $q_{i j}$. Therefore, since $q_{i i}<0$ for all $i=1,2,3,4$ under the condition of the Gersgorin theorem with all the conditions $\mu+d_{1}+r_{1}>2\left(b \theta+\beta_{1} S_{3}\right)$ and $\mu+d_{2}+r_{2}>2\left(\beta_{2} S_{3}+\beta_{3} V_{3}\right)$, it is easy to confirm that all the eigenvalues of matrix (28) have a negative real parts and then $e_{3}$ is locally asymptotically stable. Otherwise it is saddle point. 


\section{Global Stability Analysis}

Now, we investigate the global stability of all equilibrium points of the our model (5). First, we prove that of the disease free point $e_{0}$ by using CastilloChavez method for inform see [20]. The remaining three points can be prove the global stability with help of LaSalle through define the Lyapunov function for inform see [21].

Theorem 6.1: If $\mathcal{R}_{0}<1$, the $e_{0}$ of proposed model (5) is globally asymptotically stable.

Proof. Let $Y=(S, V)$ be the uninfected individuals and $Z=\left(I_{B}, I_{C}\right)$ represent to the HBV and HCV infected and $e_{0}=\left(S_{0}, V_{0}, 0,0\right)$ represents to the disease free equilibrium point. We have

$$
\frac{d X}{d t}=\mathrm{K}(\mathrm{Y}, \mathrm{Z})=\left\{\begin{array}{l}
b\left(1-\theta I_{B}\right)-\left(\mu+\epsilon+p+\beta_{1} I_{B}+\beta_{2} I_{C}\right) S+\psi V \\
\epsilon S-\left(\mu+\psi+\beta_{3} I_{C}\right) V .
\end{array}\right.
$$

If $S=S_{0}, V=V_{0}$ and $K(Y, 0)=0$, it becomes

$\frac{d X}{d t}=\left\{\begin{array}{l}b-(\mu+\epsilon) S_{0}+\psi V_{0} \\ \epsilon S_{0}-(\mu+\psi) V_{0}\end{array}\right.$

As $t \rightarrow \infty$, and $Y \rightarrow Y_{0}$. Therefore, $Y=Y_{0}=\left(S_{0}, V_{0}\right)$ is globally asymptotically stable. Now,

$$
\begin{gathered}
\mathrm{BZ}-\bar{G}(Y, Z)=\left(\begin{array}{cc}
b \theta+\beta_{1} S_{0}-\left(\mu+d_{1}+r_{1}\right) & 0 \\
0 & \beta_{2} S_{0}+\beta_{3} V_{0}-\left(\mu+d_{2}+r_{2}\right)
\end{array}\right) \cdot\left(\begin{array}{c}
I_{B} \\
I_{V}
\end{array}\right) \\
-\left(\begin{array}{c}
\beta_{1} I_{B}\left[S_{0}-S\right]+\sigma P S \\
\beta_{2} I_{C}\left[S_{0}-S\right]+\beta_{3} I_{C}\left[V_{0}-V\right]+(1-\sigma) P S
\end{array}\right) .
\end{gathered}
$$

Such that

$\mathrm{B}=\left(\begin{array}{cc}b \theta+\beta_{1} S_{0}-\left(\mu+d_{1}+r_{1}\right) & 0 \\ 0 & \beta_{2} S_{0}+\beta_{3} V_{0}-\left(\mu+d_{2}+r_{2}\right)\end{array}\right) ;$

$Z=\left(\begin{array}{l}I_{B} \\ I_{V}\end{array}\right)$

and $\bar{G}(Y, Z)=\left(\begin{array}{c}\beta_{1} I_{B}\left[S_{0}-S\right]+\sigma P S \\ \beta_{2} I_{C}\left[S_{0}-S\right]+\beta_{3} I_{C}\left[V_{0}-V\right]+(1-\sigma) P S\end{array}\right)$.

In model (5), $S_{0}+V_{0} \leq \frac{b}{\mu}$, is the bound for the population, clearly, $S, V, I_{B}, I_{C} \leq$ $\frac{b}{\mu}$. Then, $\bar{G}(Y, Z) \geq 0$. Thus, the disease free equilibrium point $e_{0}$ is globally asymptotically stable when $\mathcal{R}_{0}<1$.

Theorem 6.2: If $\mathcal{R}_{0 B} \leq 1<\mathcal{R}_{0}$, the $e_{1}$ of proposed model (5) is global asymptotically stable.

Proof. To confirm the result, we choose the Lyapunov function as follows

$$
L_{1}=\int_{S_{1}}^{S}\left(1-\frac{S_{1}}{x}\right) d x+\int_{V_{1}}^{V}\left(1-\frac{V_{1}}{x}\right) d x+I_{B}+\int_{I_{C 1}}^{I_{C}}\left(1-\frac{I_{C 1}}{x}\right) d x .
$$

The derivative of $L_{1}(t)$ corresponding to the model solutions is 


$$
\frac{d L_{1}}{d t}=\left(1-\frac{S_{1}}{S}\right) \frac{d S}{d t}+\left(1-\frac{V_{1}}{V}\right) \frac{d V}{d t}+\frac{d I_{B}}{d t}+\left(1-\frac{I_{C 1}}{I_{C}}\right) \frac{d I_{C}}{d t} .
$$

In following from direct simplify

$$
\begin{aligned}
\left(1-\frac{S_{1}}{S}\right) \frac{d S}{d t}= & \left(1-\frac{S_{1}}{S}\right)\left[b\left(1-\theta I_{B}\right)-\left(\mu+\epsilon+b+\beta_{1} I_{B}+\beta_{2} I_{C}\right) S+\psi V\right] \\
= & \left(1-\frac{S_{1}}{S}\right)\left[-\theta b I_{B}-\left(\mu+\epsilon+p+\beta_{1} I_{B}+\beta_{2} I_{C}\right) S+\psi V+\left(\mu+\epsilon+p+\beta_{2} I_{C 1}\right) S_{1}-\psi V_{1}\right] \\
= & \beta_{2} S_{1} I_{C 1}\left[1-\frac{S_{1}}{S}-\frac{S I_{C}}{S_{1} I_{C 1}}+\frac{I_{C}}{I_{C 1}}\right]+\beta_{1} S_{1} I_{B}\left[1-\frac{S}{S_{1}}-\frac{\theta b}{\beta_{1} S_{1}}+\frac{\theta b}{\beta_{S} S}\right] \\
& +(\mu+\epsilon+p) S_{1}\left[1-\frac{S_{1}}{S}-\frac{S}{S_{1}}+1\right]+\frac{\psi S_{1} V_{1}}{S}\left[1-\frac{V}{V_{1}}-\frac{s}{S_{1}}+\frac{V S}{V_{1} S_{1}}\right],
\end{aligned}
$$

$$
\begin{aligned}
\left(1-\frac{V_{1}}{V}\right) \frac{d V}{d t}= & \left(1-\frac{V_{1}}{V}\right)\left[\epsilon S-\left(\mu+\psi+\beta_{3} I_{C}\right) V\right] \\
= & \left(1-\frac{V_{1}}{V}\right)\left[\epsilon S-\left(\mu+\psi+\beta_{3} I_{C}\right) V-\epsilon S_{1}+\left(\mu+\psi+\beta_{3} I_{C 1}\right) V_{1}\right] \\
= & \beta_{3} V_{1} I_{C 1}\left[1-\frac{V_{1}}{V}-\frac{V I_{C}}{V_{1} I_{C 1}}+\frac{I_{C}}{I_{C 1}}\right]+\frac{\epsilon S_{1} V_{1}}{V}\left[1-\frac{S}{S_{1}}-\frac{V}{V_{1}}+\frac{S V}{S_{1} V_{1}}\right] \\
& +(\mu+\psi) V_{1}\left[1-\frac{V_{1}}{V}-\frac{V_{1}}{V_{1}}+1\right],
\end{aligned}
$$

$$
\frac{d I_{B}}{d t}=\left[b \theta+\beta_{1} S-\mu-d_{1}-r_{1}\right] I_{B},
$$

$$
\begin{aligned}
\left(1-\frac{I_{C 1}}{I_{C}}\right) \frac{d I_{C}}{d t}= & \left(1-\frac{I_{C 1}}{I_{C}}\right)\left[\left(\beta_{2} S+\beta_{3} V-\mu-d_{2}-r_{2}\right) I_{C}+p S\right] \\
& =\left(1-\frac{I_{C 1}}{I_{C}}\right)\left[\left(\beta_{2} S+\beta_{3} V-\mu-d_{2}-r_{2}\right) I_{C}+p S-\left(\beta_{2} S_{1}+\beta_{3} V_{1}-\mu-d_{2}-r_{2}\right) I_{C 1}-p S_{1}\right] \\
& =\frac{\beta_{2} S_{1} I_{C 1}^{2}}{I_{C}}\left[1-\frac{I_{C}}{I_{C 1}}-\frac{S I_{C}}{S_{1} I_{C 1}}+\frac{S I_{C}^{2}}{S_{1} I_{C 1}^{2}}\right]+\frac{\beta_{3} V_{1} I_{C 1}^{2}}{I_{C}}\left[1-\frac{I_{C}}{I_{C 1}}-\frac{V I_{C}}{V_{1} I_{C 1}}+\frac{V I_{C}^{2}}{V_{1} I_{C 1}^{2}}\right] \\
& +\left(\mu+d_{2}+r_{2}\right)\left[1-\frac{I_{C 1}}{I_{C}}-\frac{I_{C}}{I_{C 1}}+1\right]+\frac{p S_{1} I_{C 1}}{I_{C}}\left[1-\frac{S}{S_{1}}-\frac{I_{C}}{I_{C 1}}+\frac{S I_{C}}{S_{1} I_{C 1}}\right] .
\end{aligned}
$$

Clearly, by substituting equations (31)-(34) in to equation (30), we get

$$
\begin{aligned}
\frac{d L_{1}}{d t}=\beta_{2} & S_{1} I_{C 1}\left[1-\frac{S_{1}}{S}+\frac{I_{C}}{I_{C 1}}\left(1-\frac{S}{S_{1}}\right)\right]+\beta_{1} S_{1} I_{B}\left[1-\frac{S}{S_{1}}+\frac{\theta b}{\beta_{1} S}\left(1-\frac{S}{S_{1}}\right)\right] \\
+ & (\mu+\epsilon+p) S_{1} I_{C}\left[2-\frac{S_{1}}{S}-\frac{S}{S_{1}}\right]+\frac{\psi S_{1} V_{1}}{S}\left[1-\frac{V}{V_{1}}+\frac{V S}{V_{1} S_{1}}\left(1-\frac{S V_{1}}{S_{1} V}\right)\right] \\
& +\beta_{3} V_{1} I_{C 1}\left[1-\frac{V_{1}}{V}+\frac{I_{C}}{I_{C 1}}\left(1-\frac{V}{V_{1}}\right)\right]+\frac{\epsilon S_{1} V_{1}}{V}\left[1-\frac{V}{V_{1}}+\frac{V S}{V_{1} S_{1}}\left(1-\frac{S V_{1}}{S_{1} V}\right)\right] \\
& +(\mu+\psi) V_{1}\left[2-\frac{V_{1}}{V}-\frac{V}{V_{1}}\right]+\left(\mu+d_{2}+r_{2}\right)\left[2-\frac{I_{C 1}}{I_{C}}-\frac{I_{C}}{I_{C 1}}\right] \\
& +\frac{p S_{1} I_{C 1}}{I_{C}}\left[1-\frac{I_{C}}{I_{C 1}}+\frac{S I_{C}}{S_{1} I_{C 1}}\left(1-\frac{I_{C} S_{1}}{I_{C 1} S}\right)\right]+\left[b \theta+\beta_{1} S-\mu-d_{1}-r_{1}\right] I_{B} \\
& +\frac{\beta_{2} S_{1} I_{C 1}^{2}}{I_{C}}\left[1-\frac{I_{C}}{I_{C 1}}+\frac{S I_{C}^{2}}{S_{1} I_{C 1}^{2}}\left(1-\frac{I_{C 1}}{I_{C}}\right)\right]+\frac{\beta_{3} V_{1} I_{C 1}^{2}}{I_{C}}\left[1-\frac{I_{C}}{I_{C 1}}+\frac{V I_{C}^{2}}{V_{1} I_{C 1}^{2}}\left(1-\frac{I_{C 1}}{I_{C}}\right)\right] .
\end{aligned}
$$

Obviously, in equation (35),

$$
\begin{gathered}
{\left[1-\frac{I_{C}}{I_{C 1}}+\frac{S I_{C}^{2}}{S_{1} I_{C 1}^{2}}\left(1-\frac{I_{C 1}}{I_{C}}\right)\right] \leq 0,} \\
{\left[1-\frac{S}{S_{1}}+\frac{\theta b}{\beta_{1} S}\left(1-\frac{S}{S_{1}}\right)\right] \leq 0}
\end{gathered}
$$




$$
\begin{gathered}
{\left[1-\frac{V}{V_{1}}+\frac{V S}{V_{1} S_{1}}\left(1-\frac{S V_{1}}{S_{1} V}\right)\right] \leq 0,} \\
{\left[1-\frac{V_{1}}{V}+\frac{I_{C}}{I_{C 1}}\left(1-\frac{V}{V_{1}}\right)\right] \leq 0,} \\
{\left[b \theta+\beta_{1} S-\mu-d_{1}-r_{1}\right] I_{B} \leq 0 .}
\end{gathered}
$$

Hence, the largest invariant subset $\frac{d L_{1}}{d t}=0$, is $e_{1}$. Then, according the result of Lasalle that HBV free of model (5) is globally asymptotically stable, for $\mathcal{R}_{0 B} \leq 1<\mathcal{R}_{0}$ and it becomes unstable for $\mathcal{R}_{0 B}>1$.

Theorem 6.3: If $\mathcal{R}_{0 C} \leq 1<\mathcal{R}_{0}$, the $e_{2}$ of proposed model (5) is global asymptotically stable.

Proof. To confirm the result, we choose the Lyapunov function as follows

$$
L_{2}=\int_{S_{2}}^{S}\left(1-\frac{S_{2}}{x}\right) d x+\int_{V_{2}}^{V}\left(1-\frac{V_{2}}{x}\right) d x+\int_{I_{B 2}}^{I_{B}}\left(1-\frac{I_{B 2}}{x}\right) d x+I_{C} .
$$

The derivative of $L_{2}(t)$ corresponding to the model solutions is

$$
\frac{d L_{2}}{d t}=\left(1-\frac{S_{2}}{S}\right) \frac{d S}{d t}+\left(1-\frac{V_{2}}{V}\right) \frac{d V}{d t}+\left(1-\frac{I_{B 2}}{I_{B}}\right) \frac{d I_{B}}{d t}+\frac{d I_{C}}{d t} .
$$

In following from direct simplify

$$
\begin{aligned}
\left(1-\frac{S_{2}}{S}\right) \frac{d S}{d t}= & \left(1-\frac{S_{2}}{S}\right)\left[b\left(1-\theta I_{B}\right)-\left(\mu+\epsilon+b+\beta_{1} I_{B}+\beta_{2} I_{C}\right) S+\psi V\right] \\
= & \left(1-\frac{S_{2}}{S}\right)\left[-\theta b I_{B}-\left(\mu+\epsilon+p+\beta_{1} I_{B}+\beta_{2} I_{C}\right) S+\psi V+\theta b I_{B 2}+\left(\mu+\epsilon+p+\beta_{1} I_{B 2}\right) S_{2}-\psi V_{2}\right] \\
= & \beta_{1} S_{2} I_{B 2}\left[1-\frac{S_{2}}{S}-\frac{S I_{B}}{S_{2} I_{B 2}}+\frac{I_{B}}{I_{B 2}}\right]+(\mu+\epsilon+p)\left[1-\frac{S_{2}}{S}-\frac{S}{S_{2}}+1\right] \\
& +\frac{\psi V_{2} S_{2}}{S}\left[1-\frac{V}{V_{2}}-\frac{S}{S_{2}}+\frac{V S}{V_{2} S_{2}}\right]+b \theta I_{B 2}\left[1-\frac{S_{2}}{S}-\frac{I_{B}}{I_{B 2}}+\frac{S_{2}}{S I_{B 2}}\right] \\
& +\beta_{2} I_{C} S_{2}\left[1-\frac{S}{S_{2}}\right],
\end{aligned}
$$

$$
\begin{aligned}
\left(1-\frac{V_{2}}{V}\right) \frac{d V}{d t}= & \left(1-\frac{V_{2}}{V}\right)\left[\epsilon S-\left(\mu+\psi+\beta_{3} I_{C}\right) V\right] \\
& =\left(1-\frac{V_{2}}{V}\right)\left[\epsilon S-\left(\mu+\psi+\beta_{3} I_{C}\right) V-\epsilon S_{2}+(\mu+\psi) V_{2}\right] \\
& =\frac{\epsilon S_{2} V_{2}}{V}\left[1-\frac{S}{S_{2}}-\frac{V}{V_{2}}+\frac{S V}{S_{2} V_{2}}\right]+(\mu+\psi)\left[1-\frac{V_{2}}{V}-\frac{V}{V_{2}}+1\right] \\
& =\beta_{3} I_{C} V_{2}\left[1-\frac{V}{V_{2}}\right],
\end{aligned}
$$

$$
\begin{aligned}
\left(1-\frac{I_{B 2}}{I_{B}}\right) \frac{d I_{B}}{d t}= & \left(1-\frac{I_{B 2}}{I_{B}}\right)\left[\left(b \theta+\beta_{1} S-\mu-d_{1}-r_{1}\right) I_{B}+\sigma p S\right] \\
& =\left(1-\frac{I_{B 2}}{I_{B}}\right)\left[\left(b \theta+\beta_{1} S-\mu-d_{1}-r_{1}\right) I_{B}+\sigma p S-\left(b \theta+\beta_{1} S_{2}-\mu-d_{1}-r_{1}\right) I_{B 2}-p S_{2}\right] \\
& =\frac{\beta_{1} S_{2} I_{B 2}^{2}}{I_{B}}\left[1-\frac{I_{B}}{I_{B 2}}+\frac{S I_{B}^{2}}{S_{2} I_{B 2}^{2}}-\frac{S}{I}{ }_{B} S_{2} I_{B 2}\right]+\left(\mu+d_{1}+r_{1}\right) I_{B 2}\left[1-\frac{I_{B 2}}{I_{B}}-\frac{I_{B}}{I_{B 2}}+1\right] \\
& +\frac{p S_{2} I_{B 2}}{I_{B}}\left[1-\frac{\sigma S}{S_{2}}-\frac{I_{B}}{I_{B 2}}+\frac{\sigma S I_{B}}{S_{2} I_{B 2}}\right]+\frac{b \theta I_{B 2}^{2}}{I_{B}}\left[1-\frac{I_{B}}{I_{B 2}}-\frac{I_{B}}{I_{B 2}}+\frac{I_{B}^{2}}{I_{B 2}^{2}}\right],
\end{aligned}
$$

$$
\frac{d I_{C}}{d t}=\left[\beta_{2} S+\beta_{3} V-\mu-d_{2}-r_{2}\right],
$$


Now, by substituting equations (38)-(41) in to equation (37), we get

$$
\begin{aligned}
\frac{d L_{2}}{d t}= & \beta_{1} S_{2} I_{B 2}\left[1-\frac{S_{2}}{S}+\frac{I_{B}}{I_{B 2}}\left(1-\frac{S}{S_{2}}\right)\right]+(\mu+\epsilon+p) S_{2}\left[2-\frac{S_{2}}{S}-\frac{S}{S 2}\right] \\
& +\frac{\psi V_{2} S_{2}}{S}\left[1-\frac{S}{S_{2}}+\frac{V S}{V_{2} S_{2}}\left(1-\frac{S_{2}}{S}\right)\right]+b \theta I_{B 2}\left[1-\frac{S_{2}}{S}+\frac{S_{2 B}}{S I_{B 2}}\left(1-\frac{S}{S}\right)\right] \\
& +\beta_{2} I_{C} S_{2}\left[1-\frac{S}{S_{2}}\right]+\frac{\epsilon S_{2} V_{2}}{V}\left[1-\frac{S}{S_{2}}+\frac{V S}{V_{2} S_{2}}\left(1-\frac{S_{2}}{S}\right)\right]+\beta_{3} I_{C} V_{2}\left[1-\frac{V}{V_{2}}\right] \\
& +(\mu+\psi) V_{2}\left[2-\frac{V_{2}}{V}-\frac{V}{V_{2}}\right]+\left(\mu+d_{1}+r_{1}\right) I_{B 2}\left[2-\frac{I_{B 2}}{I_{B}}-\frac{I_{B}}{I_{B 2}}\right] \\
& +\frac{p S_{2} I_{B 2}}{I_{B}}\left[1-\frac{I_{B}}{I_{B 2}}+\frac{\sigma S I_{B}}{S_{2} I_{B 2}}\left(1-\frac{I_{B 2}}{I_{B}}\right)\right]+\left[\beta_{2} S+\beta_{3} V-\mu-d_{2}-r_{2}\right] I_{C} \\
& +\frac{\beta_{1} S_{2} I_{B 2}^{2}}{I_{B}}\left[1-\frac{I_{B}}{I_{B 2}}+\frac{S I_{B}^{2}}{S_{2} I_{B 2}^{2}}\left(1-\frac{I_{B 2}}{I_{B}}\right)\right]+\frac{b \theta I_{B 2}^{2}}{I_{B}}\left[1-\frac{I_{B}}{I_{B 2}}+\frac{I_{B}^{2}}{I_{B 2}^{2}}\left(1-\frac{I_{B 2}}{I_{B}}\right)\right] .
\end{aligned}
$$

Obviously, in equation (42),

$$
\begin{gathered}
{\left[1-\frac{S}{S_{2}}+\frac{V S}{V_{2} S_{2}}\left(1-\frac{S_{2}}{S}\right)\right] \leq 0,} \\
{\left[1-\frac{I_{B}}{I_{B 2}}+\frac{\sigma S I_{B}}{S_{2} I_{B 2}}\left(1-\frac{I_{B 2}}{I_{B}}\right)\right] \leq 0,} \\
{\left[1-\frac{V}{V_{2}}\right] \leq 0,} \\
{\left[\beta_{2} S+\beta_{3} V-\mu-d_{2}-r_{2}\right] I_{C} \leq 0 .}
\end{gathered}
$$

Thus, the largest invariant subset $\frac{d L_{2}}{d t}=0$, is $e_{2}$. Then, according the result of Lasalle that HCV free of model (5) is globally asymptotically stable, for $\mathcal{R}_{0 C} \leq 1<\mathcal{R}_{0}$ and it becomes unstable for $\mathcal{R}_{0 C}>1$.

Theorem 6.4: If $\mathcal{R}_{0}>1$, the $e_{3}$ of proposed model (5) is global asymptotically stable.

Proof. To confirm the result, we choose the Lyapunov function as follows

$$
L_{3}=\int_{S_{3}}^{S}\left(1-\frac{S_{3}}{x}\right) d x+\int_{V_{3}}^{V}\left(1-\frac{V_{3}}{x}\right) d x+\int_{I_{B 3}}^{I_{B}}\left(1-\frac{I_{B 3}}{x}\right) d x+\int_{I_{C 3}}^{I_{C}}\left(1-\frac{I_{C 3}}{x}\right) d x .
$$

The derivative of $L_{3}(t)$ corresponding to the model solutions is

$$
\frac{d L_{3}}{d t}=\left(1-\frac{S_{3}}{S}\right) \frac{d S}{d t}+\left(1-\frac{V_{3}}{V}\right) \frac{d V}{d t}+\left(1-\frac{I_{B 3}}{I_{B}}\right) \frac{d I_{B}}{d t}+\left(1-\frac{I_{C 3}}{I_{C}}\right) \frac{d I_{C}}{d t} .
$$

In following from direct calculation

$$
\begin{aligned}
\left(1-\frac{S_{3}}{S}\right) \frac{d S}{d t}=\beta_{1} S_{3} I_{B 3}\left[1-\frac{S_{3}}{S}-\frac{S I_{B}}{S_{3} I_{B 3}}+\frac{I_{B}}{I_{B 3}}\right]+\beta_{2} S_{3} I_{C 3}\left[1-\frac{S_{3}}{S}-\frac{S I_{C}}{S_{3} I_{C 3}}+\frac{I_{C}}{I_{C 3}}\right] \\
+(\mu+\epsilon+p) S_{3}\left[1-\frac{S_{3}}{S}-\frac{S}{S_{3}}+1\right]+\frac{\psi S_{3} V_{3}}{S}\left[1-\frac{V}{V_{3}}-\frac{S}{S_{3}}+\frac{S V}{S_{3} V_{3}}\right] \\
+\frac{b \theta S_{3} I_{B}}{S}\left[1-\frac{I_{B 3}}{I_{B}}-\frac{S}{S_{3}}+\frac{S_{B 3} I_{B 3}}{S_{3} I_{B}}\right],
\end{aligned}
$$




$$
\begin{gathered}
\left(1-\frac{V_{3}}{V}\right) \frac{d V}{d t}=\beta_{3} V_{3} I_{C 3}\left[1-\frac{V_{3}}{V}-\frac{V I_{C}}{V_{3} I_{C 3}}+\frac{I_{C}}{I_{C 3}}\right]+(\mu+\psi) V_{3}\left[1-\frac{V_{3}}{V}-\frac{V}{V_{3}}+1\right] \\
+\frac{\epsilon S_{3} V_{3}}{V}\left[1-\frac{S}{S_{3}}-\frac{V}{V_{3}}+\frac{S V}{S_{3} V_{3}}\right]
\end{gathered}
$$

$$
\begin{gathered}
\left(1-\frac{I_{B 3}}{I_{B}}\right) \frac{d I_{B}}{d t}=\frac{\beta_{1} S_{3} I_{B 3}^{2}}{I_{B}}\left[1-\frac{I_{B}}{I_{B 3}}-\frac{S I_{B}}{S_{3} I_{B 3}}+\frac{S I_{B}^{2}}{S_{3} I_{B 3}^{2}}\right]+\left(\mu+d_{1}+r_{1}\right) I_{B 3}\left[1-\frac{I_{B 3}}{I_{B}}-\frac{I_{B}}{I_{B 3}}+1\right] \\
+\frac{b \theta I_{B 3}^{2}}{I_{B}}\left[1-\frac{I_{B}}{I_{B 3}}-\frac{I_{B}}{I_{B 3}}+\frac{I_{B}^{2}}{I_{B 3}^{2}}\right]+\frac{p \sigma S_{3} I_{B 3}}{I_{B}}\left[1-\frac{S}{S_{3}}-\frac{I_{B}}{I_{B 3}}+\frac{S I_{B}}{S_{3} I_{B 3}}\right], \\
\left(1-\frac{I_{C 3}}{I_{C}}\right) \frac{d I_{C}}{d t}=\frac{\beta_{2} S_{3} I_{C 3}^{2}}{I_{C}}\left[1-\frac{I_{C}}{I_{C 3}}-\frac{S I_{C}}{I_{3} I_{C 3}}+\frac{S I_{C}^{2}}{S_{3} I_{C 3}^{2}}\right]+\frac{\beta_{3} V_{3} I_{C 3}^{2}}{I_{C}}\left[1-\frac{I_{C}}{I_{C 3}}-\frac{V I_{C}}{V_{3} I_{C 3}}+\frac{V I_{C}^{2}}{V_{3} I_{C 3}^{2}}\right] \\
+\left(\mu+d_{2}+r_{2}\right) I_{C 3}\left[1-\frac{I_{C 3}}{I_{C}}-\frac{I_{C}}{I_{C 3}}+1\right]+\frac{(1-\sigma) S_{3} I_{C 3}}{I_{C}}\left[1-\frac{S}{S_{3}}-\frac{I_{C}}{I_{C 3}}+\frac{S I_{C}}{S_{3} I_{C 3}}\right] .
\end{gathered}
$$

Now, by substituting equations (45)-(48) in to equation (44), we get

$$
\begin{aligned}
\frac{d L_{3}}{d t}= & \beta_{1} S_{3} I_{B 3} I_{C} I_{B}\left[1-\frac{S_{3}}{S}+\frac{I_{B}}{I_{B 3}}\left(1-\frac{S}{S_{3}}\right)\right]+\beta_{2} S_{3} I_{C 3}\left[1-\frac{S_{3}}{S}+\frac{I_{C}}{I_{C 3}}\left(1-\frac{S}{S_{3}}\right)\right] \\
& +\beta_{3} V_{3} I_{C 3}\left[1-\frac{V_{3}}{V}+\frac{I_{C}}{I_{C 3}}\left(1-\frac{V}{V_{3}}\right)\right]+\frac{\psi S_{3} V_{3}}{S}\left[1-\frac{V}{V_{3}}+\frac{S V}{S_{3} V_{3}}\left(1-\frac{V_{3}}{V}\right)\right] \\
& +\frac{\epsilon S_{3} V_{3}}{V}\left[1-\frac{V}{V}+\frac{S V}{S_{3} V_{3}}\left(1-\frac{V_{3}}{V}\right)\right]+(\mu+\epsilon+p) S_{3}\left[2-\frac{S_{3}}{S}-\frac{S}{S_{3}}\right] \\
& +(\mu+\psi) V_{3}\left[2-\frac{V_{3}}{V}-\frac{V}{V_{3}}\right]+\left(\mu+d_{1}+r_{1}\right) I_{B 3}\left[2-\frac{I_{B 3}}{I_{B}}-\frac{I_{B}}{I_{B 3}}\right] \\
& +\left(\mu+d_{2}+r_{2}\right) I_{C 3}\left[2-\frac{I_{C 3}}{I_{B}}-\frac{I_{B}}{I_{B 3}}\right]+\frac{b S_{3} I_{B}}{S}\left[1-\frac{I_{B 3}}{I_{B}}+\frac{S_{I_{3}}}{I_{3} I_{B}}\left(1-\frac{I_{B}}{I_{B 3}}\right)\right] \\
& +\frac{b \theta I_{B 3}^{2}}{I_{B}}\left[1-\frac{I_{B}}{I_{B 3}}+\frac{I_{B}^{2}}{I_{B 3}^{2}}\left(1-\frac{I_{B}}{I_{B 3}}\right)\right]+\frac{\sigma p S_{3} I_{B 3}}{I_{B}}\left[1-\frac{I_{B}}{I_{B 3}}+\frac{S I_{B}}{S_{3} I_{B 3}}\left(1-\frac{I_{B 3}}{I_{B}}\right)\right] \\
& +\frac{(1-\sigma) p S_{3} I_{C 3}}{I_{C}}\left[1-\frac{I_{C}}{I_{C 3}}+\frac{S I_{C}}{S_{3} I_{C 3}}\left(1-\frac{I_{C 3}}{I_{C}}\right)\right]+\frac{\beta_{1} S_{3} I_{B 3}^{2}}{I_{B}}\left[1-\frac{I_{B}}{I_{B 3}}+\frac{S I_{B}^{2}}{S_{3} I_{B 3}^{2}}\left(1-\frac{I_{B 3}}{I_{B}}\right)\right] \\
& +\frac{\beta_{2} S_{3} I_{C 3}^{2}}{I_{C}}\left[1-\frac{I_{C}}{I_{C 3}}+\frac{S I_{C}^{2}}{S_{3} I_{C 3}^{2}}\left(1-\frac{I_{C 3}}{I_{C}}\right)\right]+\frac{\beta_{3} V_{3} I_{C 3}^{2}}{I_{C}}\left[1-\frac{I_{C}}{I_{C 3}}+\frac{V I_{C}^{2}}{V_{3} I_{C 3}^{2}}\left(1-\frac{I_{C 3}}{I_{C}}\right)\right] .
\end{aligned}
$$

Now, in equation (49) if the following

$$
\begin{gathered}
{\left[1-\frac{S_{3}}{S}+\frac{I_{B}}{I_{B 3}}\left(1-\frac{S}{S_{3}}\right)\right] \leq 0} \\
{\left[1-\frac{I_{B}}{I_{B 3}}+\frac{S I_{B}^{2}}{S_{3} I_{B 3}^{2}}\left(1-\frac{I_{B 3}}{I_{B}}\right)\right] \leq 0} \\
{\left[1-\frac{I_{C}}{I_{C 3}}+\frac{V I_{C}^{2}}{V_{3} I_{C 3}^{2}}\left(1-\frac{I_{C 3}}{I_{C}}\right)\right] \leq 0} \\
{\left[1-\frac{V}{V_{3}}+\frac{S V}{S_{3} V_{3}}\left(1-\frac{V_{3}}{V}\right)\right] \leq 0}
\end{gathered}
$$

Then, the largest invariant subset $\frac{d L_{3}}{d t}=0$, is $e_{3}$. Then, according the result of Lasalle that endemic equilibrium point of model (5) is globally asymptotically stable, for $\mathcal{R}_{0}>1$ and it becomes unstable for $\mathcal{R}_{0}<1$. 

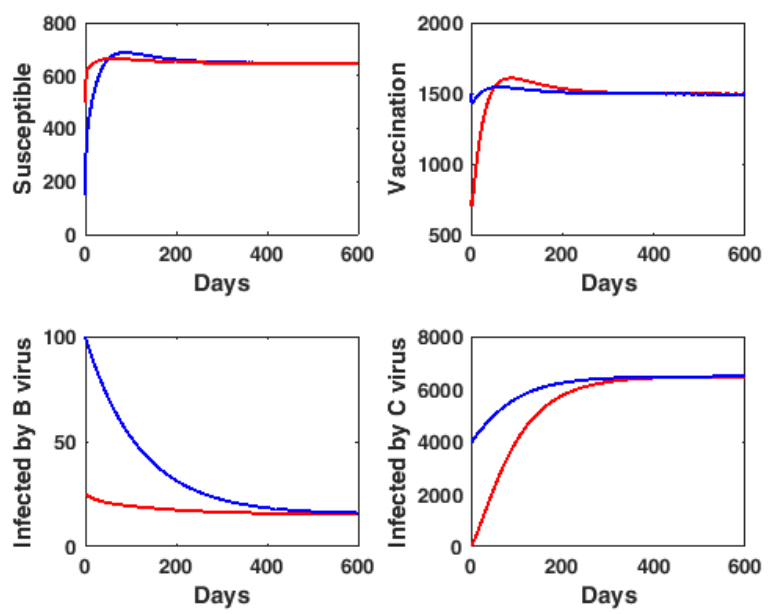

Fig. 2 The trajectory of model (5) approaches to globally asymptotically stable of endemic equilibrium point.

\section{Numerical Simulation}

To understand the influence of parameters and confirm the previous analytical results and compare, so we should investigate that through numerical simulation with choosing realistic values for parameters in this section. It is well known that the model parameters affect the dynamical behavior of the model greatly. This occurs due to either error in their measurement or missing the parameter values. Hence, the impact of varying the model's parameters on the behavior of the solution of the model is important. Therefore, the parameters that are having vital roles in the dynamics of the model will be given clear attention to understand their effects on the control of the spread of disease. We take the parameters values range adopt to some references as state in follows $b=100 ; \theta=1 \times 10^{-5} ; \beta_{1}=1 \times 10^{-6} ; \beta_{2}=1 \times 10^{-6} ; \beta_{3}=$ $1 \times 10^{-6} ; \epsilon=0.5 ; \mu=0.01 ; p=0.1 ; \psi=0.2 ; d_{1}=2 \times 10^{-5} ; d_{2}=1 \times 10^{-4} ; r_{1}=$ $1 \times 10^{-6} ; r_{2}=0.002 ; \sigma=0.002$, with time period to one year as well as two initial points $(150,750,25,15)$ is (blue line) and $(500,1500,100,4000)$ (red line). We show that the dynamic behavior of the model (5) approaches to the $e_{3}=(700,1500,16,6400)$ and $\mathcal{R}_{0}=1.4>1$, see Figure $(2)$.

However, for the same data used with take value of parameter $\sigma$, so that $\sigma=$ 1 we have, the trajectories of model (5) approaches to the $e_{2}=(650,1550,7700,0)$ and $\mathcal{R}_{0 c}=0.8<1<\mathcal{R}_{0}=1.22$, are drawn in figure (3).

On the other hand, for the same data used with take value of parameter $\sigma$, so that $\sigma=0$ we have, the trajectories of model (5) approaches to the $e_{1}=(650,1500,0,6400)$ and $\mathcal{R}_{0 b}=0.3<1<\mathcal{R}_{0}=1.22$, are drawn in figure $(4)$. 

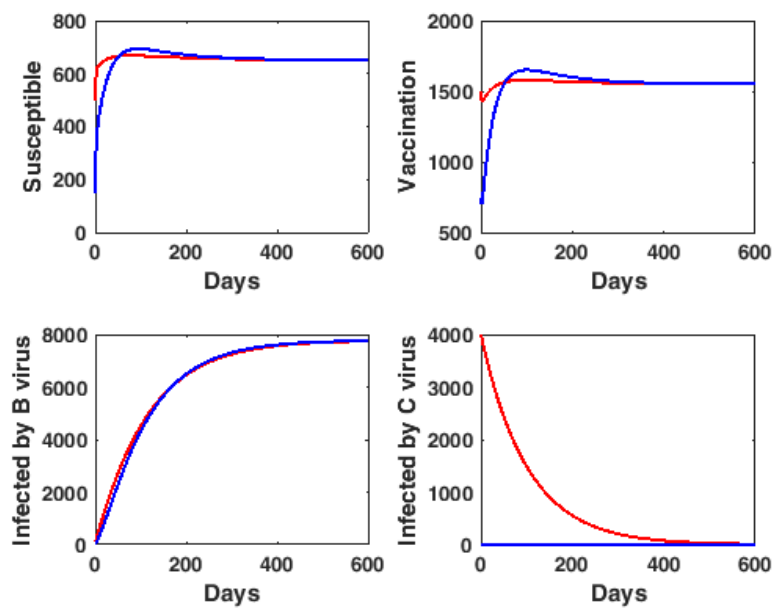

Fig. 3 The trajectory of model (5) approaches to globally asymptotically stable of hepatitis $\mathrm{C}$ virus-free equilibrium point.
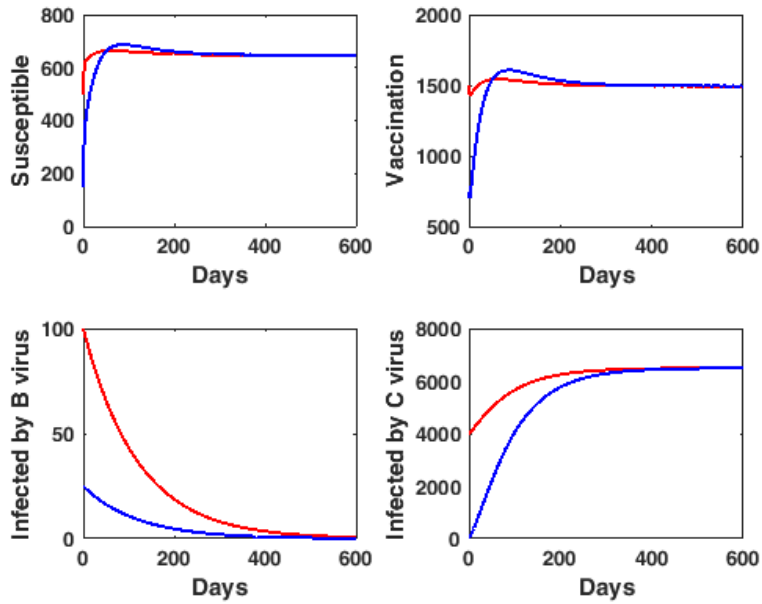

Fig. 4 The trajectory of model (5) approaches to globally asymptotically stable of hepatitis B virus-free equilibrium point.

Now, for the same data used with take values of parameters $p, \beta_{1}$ and $\beta_{3}$, so that $p=0, \beta_{1}=1 \times 10^{-7}$ and $\beta_{3}=1 \times 10^{-7}$ respectively, the trajectories of model (5) approaches to the $e_{0}=(2900,7000,0,0)$ and mathcal $R_{0}=0.431<$ 1 , are drawn in figure (5). 

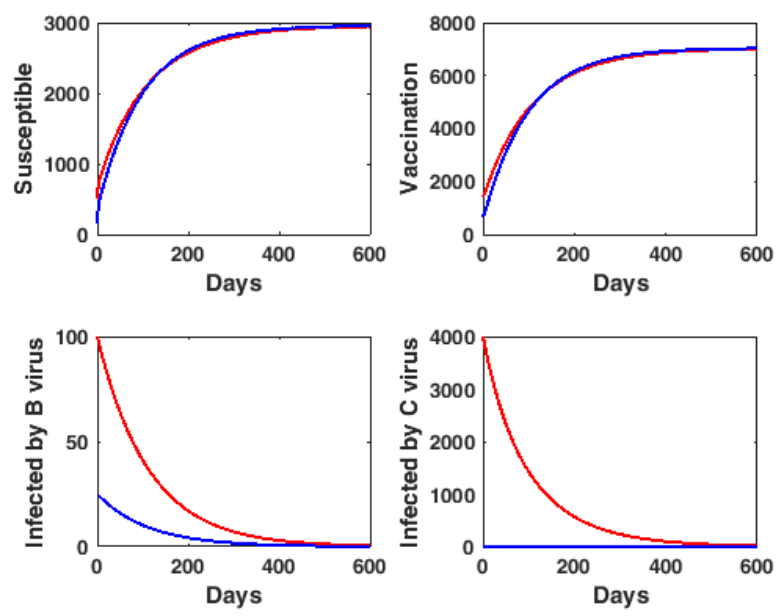

Fig. 5 The trajectory of model (5) approaches to globally asymptotically stable of diseasefree equilibrium point.

\section{Conclusion}

In this article, we have introduced a new mathematical model of hepatitis B and $\mathrm{C}$ viruses, that outbreak by into two modes: vertical (the transmission from mothers to babies) and horizontal due to the direct contact between the susceptible individuals with infected individuals. As well as, through the external sources of infection. The incidence of infection is described by linear function. According to the results in some studies the hepatitis disease incidence rate has decreased significantly and steadily in children because of the intensive and strong immunization campaign. Also, one of the goals of the World Health Organization in the year 2030 is to reduce the rate of injuries to $90 \%$ and deaths to $65 \%$. Therefore, we focused on the importance of vaccination against the disease and the need for efficiency of the vaccine. The proposed model has four equilibrium points namely disease-free equilibrium, hepatitis B virus-free equilibrium point, hepatitis C virus-free equilibrium point and endemic equilibrium. The stability analysis of the model shows that the disease-free equilibrium is globally asymptotically stable if the basic reproduction number $\mathcal{R}_{0}<1$, the condition of hepatitis $\mathrm{B}$ virus-free equilibrium point becomes global stability under the basic reproduction number for hepatitis $\mathrm{B}$ virus $\mathcal{R}_{0 B}<1$, the dynamical behavior of proposed model converges to the hepatitis $\mathrm{C}$ virus-free equilibrium point if the basic reproduction number for hepatitis $\mathrm{C}$ virus $\mathcal{R}_{0 C}<1$. The stability analysis of the model shows that the endemic equilibrium is globally asymptotically stable if the basic reproduction number is $\mathcal{R}_{0}>1$. 


\section{Conflict of interest}

The authors declare that they have no conflicts of interest.

\section{References}

1. World Health Organization. Hepatitis B. 2020. Available from: https://www.who.int/news-room/fact-sheets/detail/hepatitis-b

2. World Health Organization. Hepatitis B., Geneva, Switzerland, 2017. Available from https://www.who.int/hepatitis/publications/global-hepatitis-report2017/en/

3. McMahon B. J., Epidemiology and natural history of hepatitis B. Semin. Liver Dis 25: 3-8-(2005).

4. Lavanchy D., Hepatitis B virus eqpidemiology disease burden treatment and current and emerging prevention and control measures. J. Viral. Hepat. 11: 97-107-(2004).

5. Ullah S., Khan M., Aguilar J., Mathematical formulation of hepatitis B virus with optimal control analysis. Optim Control Appl Meth. 1-16-(2019).

6. Alrabaiah H., Safi M., DarAssi M., et al. Optomal control analysis of hepatitis B virus with treatment and vaccination. Results in Physics 19: 103-599-(2020).

7. Thornley S., Bullen C., Roberts M., Heatitis B in a high prevalence New Zealand population: a mathematical model applied to infection control policy. J. Theor. Biol. 3: 599-603-(2008)

8. Shah S., Khan M., Farooq M., et al. Fractional order model for Heatitis B virus with treatment via Atangana-Baleanu derivative. Phys. A: Stat. Mech. Appl. 15: 122-636 (2020).

9. Bachraoui M., Hattaf K., Yousfi N., Analysis of a Fractional Reaction-Diffusion HBV Model with Cure of Infected cells. Discret Dynamics in Nature and Society 15: 122 636-(2020).

10. Hattaf K., Yousfi N., Ageneralized HBV model with diffusion and two delays. Comput. Math. Appl. 69: 31-40-(2015).

11. Mohsen A., Naji R., Dynamical Analysis Within-Host and Between-Host for HIV with the Application of Optimal Control Strategy. Iraqi Journal of Science 61: 1173-1189 (2020).

12. Gul N., Bilal R., Algehyne E., et al., The dynamics of fractional order Hepatitis B virus model with asymptomatic carriers. Alexandria Engineering Journal 60: 3945-3955(2021).

13. Saad K., Alqhtani M., Gomez J., fractional study of the hepatitis C virus infection model. Resukts in Physics 19: 103-113-(2020).

14. Zao L., Ruan S., Zhang W., On the sexual transmission dynamics of hepatitis B virus in China. J. Theor. Biol. 15: 1-12-(2015).

15. Khatun Z., Islam M., Ghosh U., Mathematical modeling of hepatitis B virus infection incorporating immune responses. Sensors International 1: 1-8 (2020).

16. Din A., Li Y., Yusuf A., Delayed hepatitis B epidemic model with stochastic analysis. Chaos, Solitons and Fractals 146: 1-17-(2021).

17. Lu M., Shu Y., Huang J., Modeling homosexual and heterosexual transmissions of hepatitis B virus in China. Journal of Biological Dynamics 15: 177-194-(2021).

18. Den P., Watmough J., Reproduction number and sub-threshold endemic equilibria for compartmental models of disease transmission. J. Math. Biosci. 180: 29-48-(2002).

19. Allen L, An Introduction to mathematical Biology, Person Prentice Hall, 1 Eds. Printed in the USA, 162-163 (2007)

20. Chavez C, Blower S., Den P., et al., Mathematical Approaches for Emerging and Reemerging Infectious Diseases, An Introduction, 1 Eds., New York, NY: SpringerVerlag (2002)

21. LaSalle J, Stability theory for difference equations. In: Studies in Ordinary Differential Equations, 1 Eds., Washington, DC: Mathematical Association of America (1977). 